\title{
Surface plasmon Fourier optics
}

\author{
A. Archambault, ${ }^{1}$ T. V. Teperik, ${ }^{1,2}$ F. Marquier, ${ }^{1}$ and J.J. Greffet ${ }^{1, *}$ \\ ${ }^{1}$ Laboratoire Charles Fabry de l'Institut d'Optique, CNRS and Université Paris-Sud, \\ Campus Polytechnique, RD 128, 91127 Palaiseau cedex, France \\ ${ }^{2}$ On leave from: Institute of Radio Engineering and Electronics (Saratov Division), \\ Russian Academy of Sciences, Zelyonaya 38, 410019 Saratov, Russia
}

\begin{abstract}
Surface plasmons are usually described as surface waves with either a complex wavevector or a complex frequency. When discussing their merits in terms of field confinment or enhancement of the local density of states, controversies regularly arise as the results depend on the choice of a complex wavevector or a complex frequency. In particular, the shape of the dispersion curves depends on this choice. When discussing diffraction of surface plasmon a scalar approximation is often used. In this work, we derive two equivalent vectorial representations of a surface plasmon field using an expansion over surface waves with either a complex wavevector or a complex frequency. These representations can be used to account for propagation and diffraction of surface waves. They can also be used to discuss the issue of field confinment and local density of states as they have a non-ambiguous relation with the two dispersion relations.
\end{abstract}

PACS numbers:

\section{INTRODUCTION}

Surface plasmons have been known since the pioneering work of Ritchie in the $1950 \mathrm{~s}^{1}$. Considerable advances made in nanotechnology in recent years and the desire to control and manipulate light at nanoscale have renewed the interest in surface plasmons ${ }^{2}$. Numerical simulations and experiments have demonstrated unique properties of different plasmonic nanostructure such as extraordinary transmission ${ }^{3,4}$, guiding $5,6,7,8$, fluorescence enhancement $\frac{9,10,11,12,13}{}$, field enhancement $14,15,16$, focussing $\frac{17}{}$, superresolution $\frac{20,21,22}{}$, omnidirectional absorption ${ }^{23,25}$, coherent thermal emission ${ }^{24,25,26}$.

In this paper, we shall focus on surface plasmons propagating along flat surfaces. Propagation of surface plasmons on a flat surface perpendicular to the $\mathrm{z}$ axis is often discussed using a mode $E(z) \exp \left[i\left(K_{x} x+K_{y} y-\omega t\right)\right]$ characterized by a frequency $\omega$ and a wave vector $\mathbf{K}=$ $K_{\mathrm{x}} \hat{\mathbf{x}}+K_{\mathrm{y}} \hat{\mathbf{y}}$ parallel to the interface. However, the surface plasmon fields diffracted by edges, guided by ridges, focussed by lenses cannot be described by a simple mode. It is well-known that a finite size beam propagating in a vacuum has to be described in terms of a linear superposition of plane waves. Differents ansatz, often neglecting polarization, have been used in the litterature to address this question $\frac{5,18,27}{}$. One of the goals of this paper is to derive a rigorous representation for the surface plasmon field. Such a superposition is the equivivalent of the angular plane wave spectrum for surface plasmons It can be used to develope a framework for surface plasmon fourier optics.

In doing so, a difficulty arises. When losses are taken into account, a mode with real $\mathbf{K}$ and real $\omega$ is no longer a valid solution. Although we can still use a Fourier representation with real $\mathbf{K}$ and real $\omega$, it is not convenient to deal with waves that are not a solution. Elementary solutions using either a complex $\mathbf{K}$ or a complex $\omega$ can be found. However, we cannot assume that they form a basis. The first issue is thus to derive a general representation for the surface plasmon field as a superposition of modes. The second issue is related to the dispersion relation. A dispersion relation can be found when using either a complex $\mathbf{K}$ and a real $\omega$ or vice versa. These two choices leads to different shapes as seen in Fig 1. One dispersion relation has an asymptote for very large values of $K$ while the other has limited values of $K$ and presents a backbending.

This issue was first noted by Alexander et al $\stackrel{28}{\underline{2}}$ and later discussed by Arakawa et al $\underline{29}$. Arakawa remarked that when plotting the position of the dips in a reflectivity experiment where the angle of incidence is varied at fixed frequency, one finds the dispersion relation with backbending. Instead, when plotting the points obtained from a spectrum at fixed angle, one finds the dispersion relation without backbending. This approach seems simple and easily applicable. It is sufficient to explain the attenuated total reflection (ATR) experiments. Nevertheless, it is not a general prescription that can be used to discuss all possible issues. Let us illustrate this point by addressing two questions regarding the most important properties of surface plasmons : confinment of the fields and large density of states. For a theoretical discussion of these applications, different dispersion relations lead to different predictions. Confinment of the field is the key property regarding applications such as optical lithography, enhanced non-linear effects or superresolution issues. The dispersion relation with a backbending predicts a cut-off spatial frequency and therefore a resolution limit whereas the dispersion relation without backbending does not predict any resolution limit. Enhancement of the local density of states (or Purcell effect) is fundamental for fluorescence enhancement and more light emission assisted by surface plasmons. The dispersion relation with a backbending again predicts a cut-off spatial frequency and therefore an upper limit to the LDOS. No limit is predicted by the other dispersion 

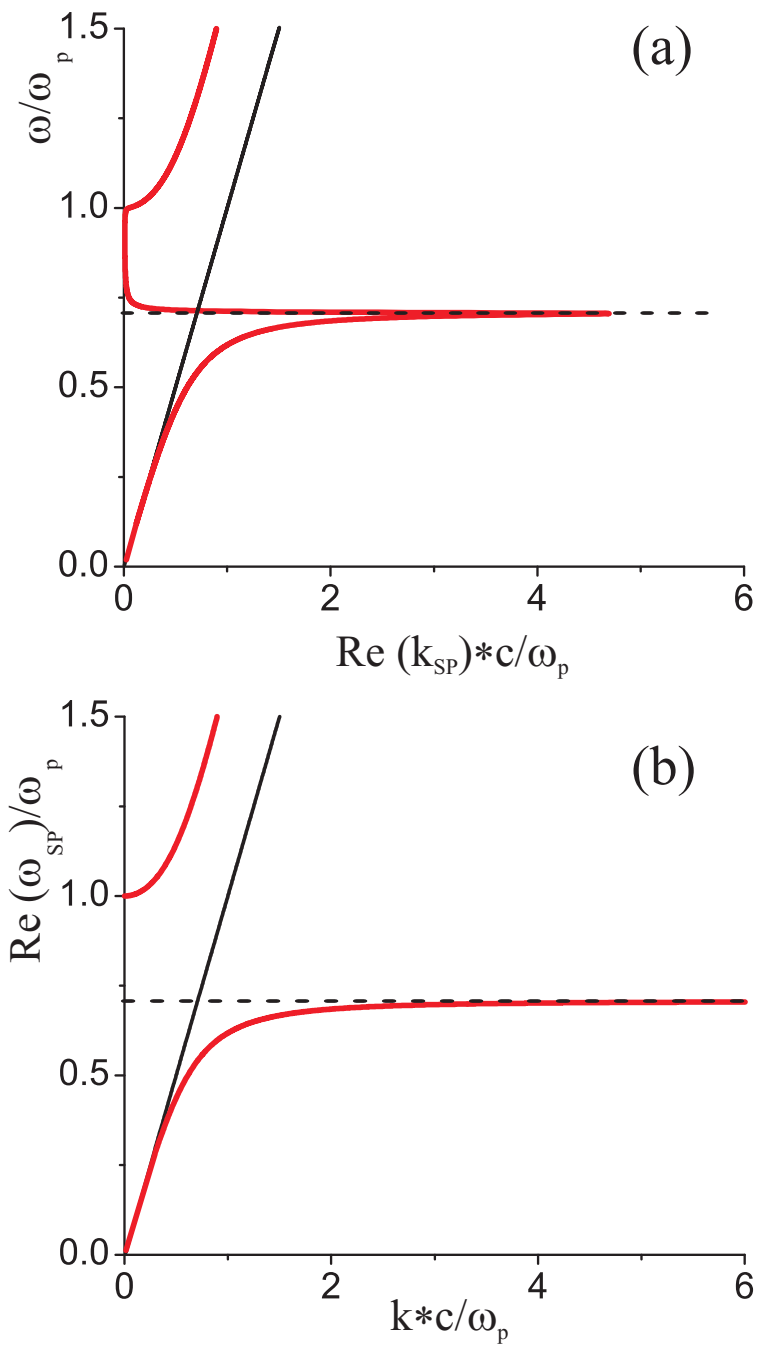

FIG. 1: (color online) Dispersion of surface plasmons propagating along the metal/air interface. (a) Real $\omega$ is chosen to obtain a complex $K_{s p}$. (b) Real $K$ is chosen to obtain a complex $\omega_{s p}$. The flat asymptote (dashed line) situated at $\omega_{p} / \sqrt{2}$ represents the nonretarded surface plasmon solution. The slanting solid line represents the light cone inside which a wave is propagating (radiative) and outside which is evanescent (surface).

relation. A general discussion on the applicability of the different dispersion relations is thus needed.

In this paper we start by deriving two general representations of the surface plasmon field in terms of linear superpositions of modes having the structure $\exp \left[i\left(K_{x} x+\right.\right.$ $\left.\left.K_{y} y+\gamma z-\omega t\right)\right]$ with a well-defined polarization. Each representation is associated with either a complex frequency or a complex wavevector and therefore to a particular dispersion relation. We then show that the most convenient choice depends on the physical problem to be discussed. We introduce a prescription that allows to choose complex or real frequency and the associated dispersion relation. We then apply our analysis to discuss the resolution limit issue and the LDOS issue. The paper is organized as follows. For the sake of completeness, we briefly summarize the derivation of the dispersion relation in the next section. The following section introduces the general representations of the surface plasmon field. We then discuss the physical content of these representations.

\section{SURFACE PLASMON DISPERSION RELATION}

Let us consider a flat metal surface $z=0$ bounded by dielectric media with dielectric constant $\varepsilon_{1}$. For convenience, we describe the dielectric response of the metal to an electric field using the local Drude model

$$
\epsilon_{2}(\omega)=1-\frac{\omega_{\mathrm{p}}^{2}}{\omega\left(\omega+i \nu_{e}\right)},
$$

where $\omega_{p}$ is the bulk plasmon frequency and $\nu_{e}$ is a phenomenological bulk electron relaxation rate. We derive the dispersion relation of surface plasmons propagating along the metal/dielectric interface.

We search a solution of Maxwell equations for an interface between two linear isotropic and local media characterized by dielectric constants $\epsilon_{m}$ where $m=1,2$ denotes medium $1(z<0)$ or $2(z>0)$. A surface wave solution has a structure $\exp \left[i\left(\mathbf{K} \cdot \mathbf{r}+\gamma_{m}|z|-\omega t\right)\right]$ with

$$
\mathbf{K}^{2}+\gamma_{m}^{2}=\epsilon_{m} \omega^{2} / c^{2}
$$

where $\gamma_{m}$ is chosen so that $\mathfrak{I m} \gamma_{m}>0$. Boundary conditions impose the continuity of the tangential components of the electric field and $\epsilon E_{z}$. It follows that a p-polarized field can exist provided that $\epsilon_{1} \gamma_{2}=-\epsilon_{2} \gamma_{1}$. One finds that a solution is given by

$$
K^{2}=\left(\frac{\omega}{c}\right)^{2} \frac{\epsilon_{1} \epsilon_{2}}{\epsilon_{1}+\epsilon_{2}} .
$$

When dealing with an interface separating a dielectric from a non lossy metal, $\frac{\epsilon_{1} \epsilon_{2}}{\epsilon_{1}+\epsilon_{2}}$ yields a unique solution to the problem. When accounting for losses in the material, $\frac{\epsilon_{1} \epsilon_{2}}{\epsilon_{1}+\epsilon_{2}}$ is a complex number so that the dispersion relation cannot be solved using real $K$ and real $\omega$. It is necessary to consider a complex frequency or a complex wavector to find roots of the equation. Let us first choose $\omega$ real. We denote $K_{s p}$ the complex root of the equation (3). Fig प(a) shows the dispersion curve obtained from the surface plasmon dispersion relation Eq. (3) when plotting $\omega$ versus $\mathfrak{R e} K_{s p}$. This curve exhibits a back-bending in the vicinity of the frequency of non-retarded surface plasmon $\omega_{p} / \sqrt{2}$. The second possible choice is to keep a real wavector $\mathbf{K}$. We denote $\omega_{s p}$ the complex root of the equation Eq. (3). Fig 1(b) shows the dispersion curve obtained when plotting $\mathfrak{R e} \omega_{s p}$ versus $K$. It is seen in Fig प(b) that this curve exhibits an asymptote for large wavevectors.

Let us make two remarks regarding the dispersion relation. We first note that Eq.(3) is also a solution of 
$\epsilon_{1} \gamma_{2}=\epsilon_{2} \gamma_{1}$ which defines a zero of the reflection factor, i.e. the Brewster angle. It can be checked that the upper branch in Fig 1(b) is not a surface wave but the locus of the Brewster angle in the $(\omega, K)$ plane. Finally, we note that a surface plasmon is a collective oscillation of charge density. When the frequency $\omega$ is smaller than $\nu_{e}$, the collective electron oscillation is overdamped. In this frequency regime, Eq.(3) describes a surface wave that has no longer the character of a surface plasmon. Thus, Eq.(3) describes the Brewster angle for $\omega>\omega_{p}$, a surface plasmon for $\omega_{p}>\omega>\nu_{e}$, a surface wave for $\nu_{e}>\omega$.

\section{GENERAL FIELD REPRESENTATIONS}

The aim of this section is to derive a general form of the surface plasmon field. To this aim, we first use the simple interface Green's tensor that yields the general form of the field for any given source distribution. We then extract the surface plasmon contribution which is defined to be the pole contribution to the Green's tensor. We will show that this procedure leads in a natural way to different representations that make use of either a complex wavevector or a complex frequency. We emphasize that both representations will describe the same electromagnetic surface plasmon field $\mathbf{E}_{s p}(x, y, z, t)$.

Let us suppose that an arbitrary source is located nearby the dielectric-metal interface. The electric field generated by the source $\mathbf{j}(\mathbf{r}, t)$ is given by the relation

$$
\mathbf{E}(\mathbf{r}, t)=-\mu_{0} \int \mathrm{d} t^{\prime} \int \mathrm{d}^{3} \mathbf{r}^{\prime} \overleftrightarrow{G}\left(\mathbf{r}, \mathbf{r}^{\prime}, t-t^{\prime}\right) \frac{\partial \mathbf{j}\left(\mathbf{r}^{\prime}, t^{\prime}\right)}{\partial t^{\prime}},
$$

where $\mu_{0}$ is vacuum permeability. A Fourier representation can be written in the form

$$
\begin{aligned}
\overleftrightarrow{G}\left(\mathbf{r}, \mathbf{r}^{\prime}, t-t^{\prime}\right)=\int \frac{\mathrm{d}^{2} \mathbf{K}}{4 \pi^{2}} \int \frac{d \omega}{2 \pi} \overleftrightarrow{g}\left(\mathbf{K}, z, z^{\prime}, \omega\right) \\
e^{i\left[\mathbf{K}\left(\mathbf{r}-\mathbf{r}^{\prime}\right)-\omega\left(t-t^{\prime}\right)\right]},
\end{aligned}
$$

Here, the integration variables $\omega$ and $K_{x}, K_{y}$ are real. The explicit form of the Green's tensor $\overleftrightarrow{g}\left(\mathbf{K}, z, z^{\prime}, \omega\right)$ in the presence of the interface is given in Appendix A. It is seen that the Fourier transform of the Green's tensor has poles given by the denominator of the Fresnel factors for $p$-polarized field. For a dielectric/metal interface, they correspond to the surface plasmon as discussed previously. Thus, the Green's tensor can be split into two terms : the pole contribution that yields the surface plasmon and the remaining contribution that yields a regularized Green's tensor.

$$
\overleftrightarrow{G}=\overleftrightarrow{G}_{r e g}+\overleftrightarrow{G}_{s p}
$$

where the pole contribution to the Green's tensor $\overleftrightarrow{G}_{s p}$ can be explicitly derived using the residue theorem. $\overleftrightarrow{G}_{r e g}$ is the contribution of the regularized Green's dyadic. It can be shown that the Green's tensor can be evaluated using a contour deformation in the complex plane and that the regularized term is essentially due to the contribution along the branch cut. This contribution is often termed cylindrical wave or creeping wave. The relative importance of these terms is well documented in classical texts for radiowaves 30.31 . The analysis of their respective contribution was of practical importance in the early days of telecommunications as radiowaves were guided by the earth. This issue has been discussed recently in the context of optics 4.33 . In this paper, we shall not pursue this discussion and focus instead on the surface wave contribution defined as the pole contribution.

$$
\mathbf{E}_{s p}(\mathbf{r}, t)=-\mu_{0} \int \mathrm{d} t^{\prime} \int \mathrm{d}^{3} \mathbf{r}^{\prime} \overleftrightarrow{G}_{s p}\left(\mathbf{r}, \mathbf{r}^{\prime}, t-t^{\prime}\right) \frac{\partial \mathbf{j}\left(\mathbf{r}^{\prime}, t^{\prime}\right)}{\partial t^{\prime}}
$$

When solving Eq.(3), we can consider that $\omega$ is real and find a complex $K_{\mathrm{sp}}$ or we can impose a real value to $K$ and find a complex root $\omega_{s p}$. Thus, when extracting the poles, it is a matter of choice to consider that they are poles in the complex frequency plane or in the complex wavevector plane. We find either a couple of poles $\omega_{s p}$ and $-\omega_{s p}^{*}$ or a complex wavevector pole $K_{s p}^{2}$ hence two poles for the component of the wavevector along the $x$ axis $K_{\mathrm{x}, \mathrm{sp}}$ and $-K_{\mathrm{x}, \mathrm{sp}}$ for a given component along the $y$ axis $K_{y}$ as $K_{s p}^{2}=K_{\mathrm{x}, \mathrm{sp}}^{2}+K_{y}^{2}$. It follows that we can cast the pole contribution to the Green's tensor in the form :

$$
\overleftrightarrow{g}_{s p}\left(\mathbf{K}, z, z^{\prime}, \omega\right)=\frac{\overleftrightarrow{f}_{\omega_{s p}}\left(\mathbf{K}, z, z^{\prime}\right)}{\omega-\omega_{s p}}+\frac{\overleftrightarrow{f}_{-\omega_{s p}^{*}}\left(\mathbf{K}, z, z^{\prime}\right)}{\omega+\omega_{s p}^{*}}
$$

where $\stackrel{\leftrightarrow}{f}_{\omega_{s p}}\left(\mathbf{K}, z, z^{\prime}\right)$ and $\overleftrightarrow{f}_{-\omega_{s p}^{*}}\left(\mathbf{K}, z, z^{\prime}\right)$ are the residues of $\overleftrightarrow{g}$ at $\omega_{s p}$ and $-\omega_{s p}^{*}$ respectively, or in the form :

$$
\begin{aligned}
& \overleftrightarrow{g}_{s p}\left(\mathbf{K}, z, z^{\prime}, \omega\right)= \\
& \quad \frac{\stackrel{\leftrightarrow}{f}_{K_{\mathrm{x}, \mathrm{sp}}}\left(K_{\mathrm{y}}, z, z^{\prime}, \omega\right)}{K_{\mathrm{x}}-K_{\mathrm{x}, \mathrm{sp}}}+\frac{\stackrel{\leftrightarrow}{f}_{-K_{\mathrm{x}, \mathrm{sp}}}\left(K_{\mathrm{y}}, z, z^{\prime}, \omega\right)}{K_{\mathrm{x}}+K_{\mathrm{x}, \mathrm{sp}}}
\end{aligned}
$$

where $\overleftrightarrow{f}_{K_{\mathrm{x}, \mathrm{sp}}}\left(K_{\mathrm{y}}, z, z^{\prime}, \omega\right)$ and $\overleftrightarrow{f}_{-K_{\mathrm{x}, \mathrm{sp}}}\left(K_{\mathrm{y}}, z, z^{\prime}, \omega\right)$ are the residues of $\overleftrightarrow{g}$ at $K_{\mathrm{x}}$, sp and $-K_{\mathrm{x}}$, sp respectively.

These two choices leads to two different forms of the surface plasmon field given by Eq.(7). We now examine these forms in detail.

\section{A. Surface plasmon field representation with a real wavevector}

In this section we derive the analytical form of the surface plasmon field using real wavevectors. For this purpose we evaluate the pole contribution to the Green's tensor by integrating in the complex $\omega$ plane. The complex pole $\omega_{s p}$ then yields a contribution for $t-t^{\prime}>0$ that varies as $\exp \left(-i \omega_{s p}\left(t-t^{\prime}\right)\right)$. After integration, we find :

$$
\begin{gathered}
\overleftrightarrow{G}_{s p}=H\left(t-t^{\prime}\right) 2 \mathfrak{R e} \int \frac{\mathrm{d}^{2} \mathbf{K}}{(2 \pi)^{2}}(-i) \stackrel{\leftrightarrow}{f}_{\omega_{s p}}\left(\mathbf{K}, z, z^{\prime}\right) \\
e^{i\left[\mathbf{K} \cdot\left(\mathbf{r}-\mathbf{r}^{\prime}\right)-\omega_{s p}\left(t-t^{\prime}\right)\right]}
\end{gathered}
$$


where $\overleftrightarrow{f}_{\omega_{s p}}\left(\mathbf{K}, z, z^{\prime}\right)$ is the residue of $\overleftrightarrow{g}$ at $\omega_{\text {sp }}$. It is given in the appendix $\mathrm{A}$. It follows from Eq. (7) that the field can be cast in the form of a linear superposition of modes with real wavevector and complex frequency :

$$
\begin{array}{r}
\mathbf{E}_{\mathrm{sp}}=2 \mathfrak{R e} \int \frac{\mathrm{d}^{2} \mathbf{K}}{(2 \pi)^{2}} E(\mathbf{K}, t)\left(\hat{\mathbf{K}}-\frac{K}{\gamma_{\mathrm{m}}} \mathbf{n}_{\mathrm{m}}\right) \\
e^{i\left(\mathbf{K} \cdot \mathbf{r}+\gamma_{\mathrm{m}}|z|-\omega_{s p} t\right)},
\end{array}
$$

where the amplitude $E(\mathbf{K}, t)$ is given in appendix $\mathrm{A}$. $\mathbf{n}_{\mathrm{m}}=-\hat{\mathbf{z}}$ if $z<0$ and $\hat{\mathbf{z}}$ if $z>0$, and $\hat{\mathbf{K}}=\mathbf{K} / K$. The surface plasmon field takes a form that looks as a mode superposition except that the amplitude $E(\mathbf{K}, t)$ depends on the time $t$. Indeed, when describing a stationary field using modes that have an exponential decay, the amplitude is necessarily time dependent. In order to obtain a superposition of modes with fixed amplitudes, it is necessary to assume that all sources are extinguished after time $t=0$ so that we observe the field after it has been excited. In that case, the decay of the mode is well described by the imaginary part of $\omega_{s p}$. Eq. (10) is thus well suited for fields excited by pulses. Note that the polarization of each mode is specified by the complex vector $\hat{\mathbf{K}}-\frac{K}{\gamma_{\mathrm{m}}} \mathbf{n}_{\mathrm{m}}$, whose component along the $z$ axis depends on the medium from which the field is evaluated.

\section{B. Surface plasmon field representation with a real frequency}

Let us now turn to the alternative choice. We consider the complex poles $K_{\mathrm{x}}$, sp and $-K_{\mathrm{x} \text {, sp }}$. The Green function can be cast in the form :

$$
\begin{aligned}
\overleftrightarrow{G_{s p}}=i \int \frac{d \omega}{2 \pi} \int \frac{\mathrm{d} K_{y}}{2 \pi} \overleftrightarrow{f}_{K_{\mathrm{x}, \mathrm{sp}}}\left(K_{y}, z, z^{\prime}, \omega\right) \\
e^{i K_{\mathrm{x}, \mathrm{sp}}\left(x-x^{\prime}\right)} e^{i K_{y}\left(y-y^{\prime}\right)} e^{-i \omega\left(t-t^{\prime}\right)}
\end{aligned}
$$

if $x-x^{\prime}>0$, and :

$$
\begin{aligned}
\overleftrightarrow{G_{s p}}=-i \int \frac{d \omega}{2 \pi} \int \frac{\mathrm{d} K_{y}}{2 \pi} \overleftrightarrow{f}_{-K_{\mathrm{x}, \mathrm{sp}}}\left(K_{y}, z, z^{\prime}, \omega\right) \\
e^{-i K_{\mathrm{x}, \mathrm{sp}}\left(x-x^{\prime}\right)} e^{i K_{y}\left(y-y^{\prime}\right)} e^{-i \omega\left(t-t^{\prime}\right)}
\end{aligned}
$$

if $\quad x-x^{\prime}<0 . \quad \overleftrightarrow{f}_{K_{\mathrm{x}, \mathrm{sp}}}\left(K_{y}, z, z^{\prime}, \omega\right)$ and $\overleftrightarrow{f}_{K-\mathrm{x}, \mathrm{sp}}\left(K_{y}, z, z^{\prime}, \omega\right)$ are the residues of $\overleftrightarrow{g}$ at $K_{\mathrm{x}, \mathrm{sp}}$ and $-K_{\mathrm{x}}$, sp. They are given in appendix $\mathrm{A}$ When inserting this form in Eq.(7), we again obtain a form for the field that is a superposition of modes whose amplitude depends on $x$ :

$$
\begin{array}{r}
\mathbf{E}=\int \frac{\mathrm{d} \omega}{2 \pi} \int \frac{\mathrm{d} K_{\mathrm{y}}}{2 \pi}\left[E_{>}\left(K_{\mathrm{y}}, \omega, x\right)\left(\hat{\mathbf{K}}^{+}-\frac{K_{s p}}{\gamma_{\mathrm{m}}} \mathbf{n}_{\mathrm{m}}\right)\right. \\
e^{i\left(K_{\mathrm{x}, \mathrm{sp}} x+K_{\mathrm{y}} y+\gamma_{\mathrm{m}}|z|-\omega t\right)} \\
+E_{<}\left(K_{\mathrm{y}}, \omega, x\right)\left(\hat{\mathbf{K}}^{-}-\frac{K_{s p}}{\gamma_{\mathrm{m}}} \mathbf{n}_{\mathrm{m}}\right) \\
\left.e^{i\left(-K_{\mathrm{x}, \mathrm{sp}} x+K_{\mathrm{y}} y+\gamma_{\mathrm{m}}|z|-\omega t\right)}\right]
\end{array}
$$

where $\hat{\mathbf{K}}^{+}=\left(K_{\mathrm{x}, \mathrm{sp}} \hat{\mathbf{x}}+K_{\mathrm{y}} \hat{\mathbf{y}}\right) / K_{s p}$ and $\hat{\mathbf{K}}^{-}=$ $\left(-K_{\mathrm{x}, \mathrm{sp}} \hat{\mathbf{x}}+K_{\mathrm{y}} \hat{\mathbf{y}}\right) / K_{s p}$. The amplitudes $E_{>}\left(K_{\mathrm{y}}, \omega, x\right)$ and $E_{<}\left(K_{\mathrm{y}}, \omega, x\right)$ are given in appendix $\mathrm{A}$. Again, it seems natural to have amplitudes of the modes that depend on $x$ if one describes a homogeneous field using modes with a decay along $x$. A proper mode representation should use only fixed amplitudes. This is possible if all the sources lie in the $x<0$ region and the region of interest is the $x>0$ region. We then obtain a surface plasmon field that can be cast in the form :

$$
\begin{array}{r}
\mathbf{E}=\int \frac{\mathrm{d} \omega}{2 \pi} \int \frac{\mathrm{d} K_{\mathrm{y}}}{2 \pi}\left(\hat{\mathbf{K}}-\frac{K_{s p}}{\gamma_{\mathrm{m}}} \mathbf{n}_{\mathrm{m}}\right) E_{>}\left(K_{\mathrm{y}}, \omega\right) \\
e^{i\left(\mathbf{K} \cdot \mathbf{r}+\gamma_{\mathrm{m}}|z|-\omega t\right)}
\end{array}
$$

where $\mathbf{K}=K_{\mathrm{x}, \mathrm{sp}} \hat{\mathbf{x}}+K_{\mathrm{y}} \hat{\mathbf{y}}$ is complex and $\hat{\mathbf{K}}=\mathbf{K} / K_{s p}$. We conclude that stationary monochromatic fields with a finite size are well described by a representation that uses complex wavevectors and real frequencies. This equation is one of the main result of this paper. Indeed, it provides a framework to develop surface plasmon Fourier optics. Similar representations have been postulated as ansatz to surface plasmons interferences 27 , propagation along a stripe $\mathrm{e}^{\frac{5}{}}$ or focussing 18 . The framework introduced above provides a rigorous derivation of the form of the surface plasmon field valid in a region with no sources. Let us emphasize that this representation is well suited to discuss propagation for $x>x_{0}$ of a surface plasmon field known along a line $x=x_{0}$. It is seen on Eq.(14) that propagation over a distance $d$ amounts to multiply each mode by a factor $\exp \left(i K_{x} d\right)$. In general, this involves modifying both the phase and the amplitude of the mode. Thus, it allows to discuss any surface wave diffraction problem. Finally, let us stress that this representation is valid for a complex wavevector $\mathbf{K}$ and a real frequency $\omega$ so that this representation is necessarily associated with a dispersion relation with backbending.

To summarize, we have shown that the surface plasmon field can be represented using modes that have either a complex frequency or a complex wavevector. However, the amplitudes may still depend on either time or space. In the case of a field excited by a pulse, the representation that uses a complex frequency is well suited. It is associated with the dispersion relation without backbending. In the case of a stationary monochromatic excitation localized in space, a representation using modes with complex wavevectors is well suited. It corresponds to a dispersion relation with backbending. This simple analysis yields a simple prescription to choose the proper dispersion relation. Note that in the case of pulses limited in space, both representations can be used.

\section{Surface plasmon field generated by a dipole}

For many applications, it is useful to know the field generated by a dipole. For instance, when considering the field scattered by a subwavelength particle, the source 


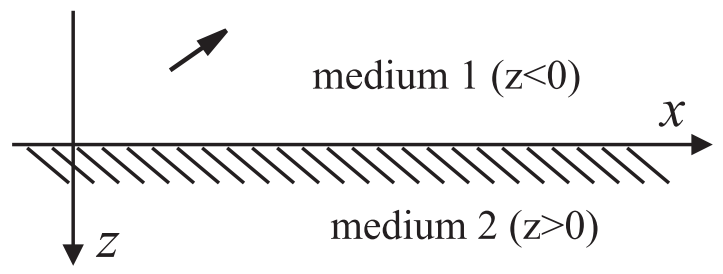

FIG. 2: A point-like dipole located nearby the dielectricmetal interface

can be represented by an electric dipole. In addition, any source can be decomposed as linear superposition of dipolar sources. Here, we derive the surface plasmon field generated by a monochromatic point-like dipole characterized by its dipole moment $\mathbf{p}_{\mathbf{0}}$ (see Fig. 2). Note that there are other contributions to the field generated by the dipole at a distance typically smaller than a wavelength. The surface plasmon contribution is typically dominant for larger distances 32,33 .

For a vertical dipole $\mathbf{p}_{\mathbf{0}} e^{-i \omega_{0} t}=p_{0} e^{-i \omega_{0} t} \hat{\mathbf{z}}$ located at a distance $d$ below the interface, at $x=y=0$, we obtain, using the cylindrical basis $\hat{\rho}, \hat{\theta}, \hat{\mathbf{z}})$ :

$$
\begin{gathered}
\mathbf{E}_{\mathrm{m}}=2 \mathfrak{R e}\left\{\left[H_{1}^{(1)}\left(K_{s p} \rho\right) \hat{\rho}+i \frac{K_{s p}}{\gamma_{m}} H_{0}^{(1)}\left(K_{s p} \rho\right) \mathbf{n}_{m}\right]\right. \\
\left.M\left(K_{s p}, \omega_{0}\right) \frac{1}{\epsilon_{0}}\left(-i \frac{K_{s p}}{\gamma_{1}}\right) p_{0} e^{i \gamma_{1} d} e^{i \gamma_{m}|z|} e^{-i \omega_{0} t}\right\}
\end{gathered}
$$

where $H_{0}^{(1)}$ and $H_{1}^{(1)}$ are Hankel functions of the first kind of zero-th and first order respectively, $K_{s p}$ is complex and verifies Eq. (3) with $\omega=\omega_{0}, M\left(K_{s p}, \omega_{0}\right)$ is given in appendix $B$ and the other notations are defined above. The details of the calculation are given in appendix $B$. Using the asymptotic forms of the Hankel functions, we obtain for the field of a vertical dipole, for $\rho$ greater than a few $1 /\left|K_{s p}\right|$ :

$$
\begin{array}{r}
\mathbf{E}_{\mathrm{m}}=2 \mathfrak{R e}\left[\frac{e^{i K_{s p} \rho}}{\sqrt{K_{s p} \rho}}\left(\hat{\rho}-\frac{K_{s p}}{\gamma_{m}} \mathbf{n}_{m}\right) M_{v}^{\prime}\left(K_{s p}, \omega_{0}\right) p_{0}\right. \\
\left.e^{i \gamma_{1} d} e^{i \gamma_{m}|z|} e^{-i \omega_{0} t}\right]
\end{array}
$$

where $M_{v}^{\prime}\left(K_{s p}, \omega_{0}\right)$ is given in appendix B Thus the surface plasmon field is analogous to a damped cylindrical wave $\frac{e^{i K_{s p} \rho}}{\sqrt{K_{s p} \rho}}$ with a polarization vector $\hat{\mathbf{r}}-\frac{K_{s p}}{\gamma_{m}} \mathbf{n}_{m}$. For a dipole oriented along the $x$ axis $\mathbf{p}_{0} e^{-i \omega_{0} t}=p_{0} e^{-i \omega_{0} t} \hat{\mathbf{x}}$, we obtain :

$$
\begin{gathered}
\mathbf{E}_{\mathrm{m}}=2 \mathfrak{R e}\left\{\left[\left(\left[H_{1}^{(1)}\left(K_{s p} \rho\right)\right]^{\prime} \hat{\rho}-i \frac{K_{s p}}{\gamma_{m}} H_{1}^{(1)}\left(K_{s p} \rho\right) \mathbf{n}_{m}\right)\right.\right. \\
\left.\cos \theta-\frac{H_{1}^{(1)}\left(K_{s p} \rho\right)}{K_{s p} \rho} \hat{\theta} \sin \theta\right] \\
\left.M\left(K_{s p}, \omega_{0}\right) \frac{1}{\epsilon_{0}} p_{0} e^{i \gamma_{1} d} e^{i \gamma_{m}|z|} e^{-i \omega_{0} t}\right\}
\end{gathered}
$$

where $\theta=(\hat{\mathbf{x}}, \hat{\rho})$. Using the asymptotic forms of the Hankel functions, we obtain :

$$
\begin{aligned}
\mathbf{E}_{\mathrm{m}}=2 \Re \mathfrak{R e}\left[\frac{e^{i K_{s p} \rho}}{\sqrt{K_{s p} \rho}}\left(\hat{\rho}-\frac{K_{s p}}{\gamma_{m}} \mathbf{n}_{m}\right) \cos \theta\right. \\
\left.M_{h}^{\prime}\left(K_{s p}, \omega_{0}\right) p_{0} e^{i \gamma_{1} d} e^{i \gamma_{m}|z|} e^{-i \omega_{0} t}\right]
\end{aligned}
$$

where $M_{h}^{\prime}\left(K_{s p}, \omega_{0}\right)$ is given in appendix B. The surface plasmon field is analogous to a damped cylindrical wave $\frac{e^{i K_{s p} \rho}}{\sqrt{K_{s p} \rho}}$ with the same polarization vector $\hat{\rho}-\frac{K_{s p}}{\gamma_{m}} \mathbf{n}_{m}$, times a factor $\cos \theta$, making the field vanishing in the direction perpendicular the dipole (here the $y$ direction) and more intense in the dipole's direction (here the $x$ direction).

\section{DISCUSSION}

In this section, we revisit two fundamental issues in the field of surface plasmons : field confinment and large density of states. Confinment and superresolution are related to the existence of wavevectors with a modulus much larger than $\omega / c$. In this respect the choice of the proper dispersion relation plays a key role as one has a cut-off wavevector whereas the other predicts no limit for the dispersion relation. Is there a limit to the resolution? Is there a limit to the local density of states ?

\section{A. Super resolution}

Let us first discuss the issue of resolution when imaging with a surface plasmon driven at frequency $\omega$ by an external source. Recent experiments on far-field optical microscopy ${ }^{21}$ launched a debate ${ }^{34,35}$ about the role of surface plasmons in super-resolution imaging effects. In Ref. 21] the dispersion curve with the asymptotic behaviour has been invoked to stress the role of surface plasmons in the image formation with nano-resolution. The resolution was estimated to be $\lambda_{s p} / 2$. Therefore, if the dispersion curve with the asymptotic behaviour is chosen, there seems to be no diffraction limit and only the amplitude decay of surface plasmon due to Ohmic losses in the metal limits the resolution. The effect of the back-bending of surface plasmon dispersion discussed in Ref. 35] limits the surface plasmon wavelength $2 \pi / \mathfrak{R e} K_{s p}$ and therefore, the resolution. Clearly, both dispersion relations do not lead to the same conclusion and a prescription to choose one or the other is needed. Let us consider a situation where a surface plasmon is excited locally by a stationary monochromatic field. From section 3, we know that it is valid to use a representation with fixed amplitudes using modes with complex wavevectors and real frequencies. This implies that the dispersion relation with real frequency (with backbending) is relevant. It follows that there is a cut-off spatial 
frequency. Indeed, as $K_{x}$ may be complex, the propagation term $\exp \left(i K_{x} x\right)$ introduces damping. In the case of a lossy medium, damping may be due to losses. However, even for a non-lossy medium ( $K_{s p}$ is real), $K_{x}=\left(K_{s p}^{2}-K_{y}^{2}\right)^{1 / 2}$ can be imaginary. This occurs when $K_{y}$ exceeds the value $K_{s p}$. This situation is the 2D analog of the evanescent waves with wavevector $K$ larger than $\omega / c$ that cannot propagate in a vacuum. Clearly, $K_{s p}$ is a cut-off frequency and the propagation term $\exp \left(i K_{x} x\right)$ works as a low-pass filter that prevents propagation of fields associated with spatial frequencies larger than $K_{s p}$. When dealing with lossy media, it is the real part of $K_{s p}$ that specifies the cut-off spatial frequency. It is seen in Fig प that it is limited by the backbending of the dispersion relation.

In summary, when discussing imaging using stationary monochromatic surface plasmons, the relevant representation is based on modes with a complex wavector and a real frequency given by Eq. (14). This corresponds to a dispersion relation that has a backbending. It follows that the resolution is limited by the cut-off spatial frequency given by the maximum value of $\mathfrak{R e} K_{s p}$.

\section{B. Local density of states}

Let us now discuss the Local Density of States (LDOS). The density of states (DOS) is a quantity that plays a fundamental role in many domains. In particular, it allows to derive all thermodynamic properties of a system. In the case of an interface, the surface modes are confined close to the interface so that it is useful to introduce the Local Density of States (LDOS) that depends on the distance to the interface $\frac{36,37}{3}$. It allows to account for the huge increase of energy density close to an interface when surface waves are excited 37,38 . It also plays a key role in defining the lifetime of a single emitter close to an interface $39,40,41,42,43$. In this context, the increase of the projected LDOS is usually normalized by the LDOS in a homogeneous medium (e.g. a vacuum) yielding the so-called Purcell factor. It is well-known in solid state physics that the density of states can be derived from the dispersion relation. More specifically, the DOS increases at a frequency $\omega$ when the dispersion relation is flat at that particular frequency. A quick look at Fig 1 shows that different dispersion relations seem to predict different LDOS. While Fig $1(\mathrm{~b})$ predicts a very large peak at $\omega_{s p} / \sqrt{2}$ due to the asymptote and no states above this frequency, Fig प(a) predicts a smaller peak and a non zero LDOS between $\omega_{s p} / \sqrt{2}$ and $\omega_{s p}$. Again, we see that a prescription is needed to choose the right dispersion relation.

A standard procedure to derive the DOS in the reciprocal space is based on the periodic boundary conditions. Assuming a surface of side $L$, the wavevector takes the form $\mathbf{K}=n_{x} \frac{2 \pi}{L} \hat{\mathbf{x}}+n_{y} \frac{2 \pi}{L} \hat{\mathbf{y}}$. In the plane $K_{x}, K_{y}$, a mode has an area $4 \pi^{2} / L^{2}$. It follows that the number of modes per unit area in $d^{2} \mathbf{K}$ is given by $d^{2} \mathbf{K} / 4 \pi^{2}$. When performing this analysis, both $K_{x}$ and $K_{y}$ are real. Thus the relevant representation uses real wavevectors and complex frequencies. The corresponding dispersion relation has no backbending and therefore presents a singularity. This is in agreement with another approach of the LDOS based on the use of the Green's tensor that predicts an asymptotic behaviour proportional to $1 /\left(z^{3}|\epsilon+1|^{2}\right)^{36}, 37$. Of course, this divergence is non physical. It is related to the modelling of the medium using a continuous description of the metal. This model cannot be valid on an atomic scale. Before reaching the atomic scale, non-local effects must be taken into account.

\section{CONCLUSION}

The purpose of this work is to clarify several issues regarding surface plasmons on flat surfaces. The first issue deals with the mode representation of the surface plasmon field. We have shown that a surface plasmon field can be represented as a sum of modes with either a complex wavevector or a complex frequency. We have shown that a representation using complex frequencies is well adapted to fields excited by pulses and that a representation using complex wavevectors is well adapted to stationary monochromatic fields excited in a finite area. The latter representation provides a rigorous formula that can be used to analyse the diffraction of a stationary surface plasmon field. This should be very useful in order to develop a surface plasmon Fourier optics framework. This formula clearly shows that the maximum value of $\mathfrak{R e} K_{s p}$ is a cut-off spatial frequency that gives an upper limit to the resolution or confinment that can be obtained using surface plasmons. As a by product, we have derived the form of the surface plasmon excited by a dipole located below the interface. Finally, we have discussed how to choose the dispersion relation (with or without backbending) depending on the issue. To illustrate this procedure, we have shown that there is a resolution limit given by the maximum value of the wavevector at the backbending point. We have also shown that the local density of states should be analysed using the dispersion relation with a real wavevector. This yields a LDOS that diverges close to the interface in agreement with the result obtained from the Green's tensor approach.

\section{APPENDIX A: CALCULATIONS OF THE SURFACE PLASMON FIELD}

For the plane interface system, it is convenient to use the representation due to $\mathrm{Sipe}_{\underline{44}}$ that consists of a decomposition over elementary plane waves. We consider the interface such as the lower medium $z<0$ is denoted as medium 1 and the upper medium $z>0$ is medium 2. We use the dyadic notation for the tensor. For instance, the $s$-component of the electric field is given by 
$\hat{\mathbf{s}} \hat{\mathbf{s}} \mathbf{E}=\hat{\mathbf{s}}(\hat{\mathbf{s}} \cdot \mathbf{E})$. Using the notations of Eq. (5), we have for the field in the lower half-space $z<0$ and currents below $z\left(z^{\prime}<z\right)$ :

$$
\begin{aligned}
& \overleftrightarrow{g}\left(\mathbf{K}, z, z^{\prime}, \omega\right)=\overleftrightarrow{g}_{0}\left(\mathbf{K}, z, z^{\prime}, \omega\right) \\
& \quad+\frac{i}{2 \gamma_{1}}\left[\hat{\mathbf{s}} r_{s} \hat{\mathbf{s}}+\hat{\mathbf{p}}_{1}^{-} r_{p} \hat{\mathbf{p}}_{1}^{+}\right] e^{-i \gamma_{1} z^{\prime}} e^{-i \gamma_{1} z}
\end{aligned}
$$

where $\overleftrightarrow{g}_{0}\left(\mathbf{K}, z, z^{\prime}, \omega\right)$ denotes the Fourier transform of the Green's tensor of an infinite space filled by medium 1 .

For the field in the upper half-space $(z>0)$ and currents still in the lower half-space $\left(z^{\prime}<0\right)$, one has :

$$
\overleftrightarrow{g}\left(\mathbf{K}, z, z^{\prime}, \omega\right)=\frac{i}{2 \gamma_{1}}\left[\hat{\mathbf{s}} t_{s} \hat{\mathbf{s}}+\hat{\mathbf{p}}_{2} t_{p} \hat{\mathbf{p}}_{1}^{+}\right] e^{-i \gamma_{1} z^{\prime}} e^{i \gamma_{2} z}
$$

The Fresnel reflection and transmission factors are given by :

$$
\begin{aligned}
r_{s} & =\frac{\gamma_{1}-\gamma_{2}}{\gamma_{1}+\gamma_{2}} & r_{p} & =\frac{\gamma_{1} \epsilon_{2}-\gamma_{2} \epsilon_{1}}{\gamma_{1} \epsilon_{2}+\gamma_{2} \epsilon_{1}} \\
t_{s} & =\frac{2 \gamma_{1}}{\gamma_{1}+\gamma_{2}} & t_{p} & =\frac{2 \gamma_{1} \sqrt{\epsilon_{1}} \sqrt{\epsilon_{2}}}{\gamma_{1} \epsilon_{2}+\gamma_{2} \epsilon_{1}}
\end{aligned}
$$

$\gamma_{m}=\sqrt{\epsilon_{m}\left(\frac{\omega}{c}\right)^{2}-K^{2}}$ for $m=1,2$ is chosen so that $\mathfrak{I m} \gamma_{m}>0$. This way, using $\epsilon_{m}\left(-\omega^{*}\right)=\epsilon_{m}^{*}(\omega)$, one has $\gamma_{m}\left(K^{*},-\omega^{*}\right)=-\gamma_{m}^{*}(K, \omega)$. The square roots of dielectric constants $\sqrt{\epsilon_{m}}$ are chosen so that $\mathfrak{R e} \sqrt{\epsilon_{m}} \geqslant 0$. $\hat{\mathbf{s}}=\mathbf{K} \times \mathbf{z} / K, \hat{\mathbf{p}}_{1}^{ \pm}=\left(K \mathbf{z} \mp \gamma_{1} \mathbf{K} / K\right) /\left(k_{0} \sqrt{\epsilon_{1}}\right)$ and $\hat{\mathbf{p}}_{2}=$ $\left(K \mathbf{z}-\gamma_{2} \mathbf{K} / K\right) /\left(k_{0} \sqrt{\epsilon_{2}}\right), k_{0}=\omega / c$ and $K=\sqrt{K_{x}^{2}+K_{y}^{2}}$ is chosen so that $\mathfrak{I m} K>0$ or $\mathfrak{I m} K=0$ and $\mathfrak{R e} K>0$. This way, one has $\hat{\mathbf{p}}\left(-\mathbf{K},-\omega^{*}\right)=-\hat{\mathbf{p}}(\mathbf{K}, \omega)^{*}$ when $\mathbf{K}$ is real and $\omega$ complex and $\hat{\mathbf{p}}\left(-\mathbf{K}^{*},-\omega\right)=\hat{\mathbf{p}}(\mathbf{K}, \omega)^{*}$ when $\mathbf{K}$ is complex and $\omega$ real.

We extend the definition of $\overleftrightarrow{g}\left(\mathbf{K}, z, z^{\prime}, \omega\right)$ to complex values of $\omega$ and assume that the denominator of the Fresnel coefficients $r_{p}$ and $t_{p}$ (whose nullity is equivalent to Eq. (3) ) has two roots $\omega_{s p}$ and $-\omega_{s p}^{*}$ :

$$
\frac{1}{\gamma_{1} \epsilon_{2}+\gamma_{2} \epsilon_{1}}=\frac{C(K, \omega)}{\left(\omega-\omega_{s p}\right)\left(\omega+\omega_{s p}^{*}\right)}
$$

with $\mathfrak{I m} \omega_{s p}<0$. $\overleftrightarrow{g}$ then features two poles at $\omega_{s p}$ and $-\omega_{s p}^{*}$. The residues of $\overleftrightarrow{g}$ at these poles can be calculated with $\overleftrightarrow{f}_{\widetilde{\omega}}\left(\mathbf{K}, z, z^{\prime}\right)=\lim _{\omega \rightarrow \widetilde{\omega}}\left[(\omega-\widetilde{\omega}) \overleftrightarrow{g}\left(\mathbf{K}, z, z^{\prime}, \omega\right)\right]$ where $\widetilde{\omega}$ denotes $\omega_{s p}$ or $-\omega_{s p}^{*}$. It comes :

$$
\begin{array}{r}
\stackrel{\leftrightarrow}{f}_{\omega_{s p}}\left(\mathbf{K}, z, z^{\prime}\right)=i \frac{\gamma_{1} \epsilon_{2}}{k_{0} \sqrt{\epsilon_{1}}} \frac{C\left(K, \omega_{s p}\right)}{2 \mathfrak{R e} \omega_{s p}}\left(\hat{\mathbf{K}}-\frac{K}{\gamma_{m}} \mathbf{n}_{m}\right) \hat{\mathbf{p}}_{1}^{+} \\
e^{-i \gamma_{1} z^{\prime}} e^{i \gamma_{m}|z|}
\end{array}
$$

where $\mathbf{n}_{m}$ denotes $-\hat{\mathbf{z}}$ for $z<0$ and $\hat{\mathbf{z}}$ for $z>0, \gamma_{m}$ denotes $\gamma_{1}$ for $z<0$ and $\gamma_{2}$ for $z>0$. $\gamma_{1}$ and $\gamma_{2}$ depends on $K$ and $\omega_{s p}$ and $\hat{\mathbf{p}}_{1}^{+}$on $\mathbf{K}$ and $\omega_{s p}$.

Using $\epsilon_{m}\left(-\omega^{*}\right)=\epsilon_{m}^{*}(\omega), \gamma_{m}\left(K,-\omega^{*}\right)=-\gamma_{m}^{*}(K, \omega)$, and $\hat{\mathbf{p}}_{1}^{+}\left(\mathbf{K},-\omega^{*}\right)=-\hat{\mathbf{p}}_{1}^{+}(-\mathbf{K}, \omega)^{*}$, we have :

$$
\stackrel{\leftrightarrow}{f}_{-\omega_{s p}^{*}}\left(\mathbf{K}, z, z^{\prime}\right)=-\overleftrightarrow{f}_{\omega_{s p}}^{*}\left(-\mathbf{K}, z, z^{\prime}\right)
$$

Using Eq. (77, (9) and (A6), one can find the amplitudes in Eq. (10) :

$$
\begin{array}{r}
E(\mathbf{K}, t)=-\mu_{0} \frac{\gamma_{1} \epsilon_{2}}{k_{0} \sqrt{\epsilon_{1}}} \frac{C\left(K, \omega_{s p}\right)}{2 \Re \mathfrak{R e} \omega_{s p}} \int \mathrm{d}^{2} \mathbf{r}^{\prime} e^{-i \mathbf{K} \cdot \mathbf{r}^{\prime}} \\
\int_{-\infty}^{0} \mathrm{~d} z^{\prime} e^{-i \gamma_{1} z^{\prime}} \int_{-\infty}^{t} \mathrm{~d} t^{\prime} e^{i \omega_{s p} t^{\prime}} \hat{\mathbf{p}}_{1}^{+} \cdot \frac{\partial \mathbf{j}}{\partial t^{\prime}}\left(\mathbf{r}^{\prime}, t^{\prime}\right)
\end{array}
$$

We now extend the definition of $\overleftrightarrow{g}\left(\mathbf{K}, z, z^{\prime}, \omega\right)$ to complex values of $K_{x}$ and assume ${ }^{45}$ that the denominator of the Fresnel coefficients $r_{p}$ and $t_{p}$ has two roots $K_{\mathrm{x}, \mathrm{sp}}=\sqrt{K_{s p}^{2}-K_{\mathrm{y}}^{2}}$ and $-K_{\mathrm{x}, \mathrm{sp}}$ :

$$
\frac{1}{\gamma_{1} \epsilon_{2}+\gamma_{2} \epsilon_{1}}=\frac{\gamma_{1} \epsilon_{2}-\gamma_{2} \epsilon_{1}}{\epsilon_{1}^{2}-\epsilon_{2}^{2}} \frac{1}{\left(K_{\mathrm{x}}-K_{\mathrm{x}, \mathrm{sp}}\right)\left(K_{\mathrm{x}}+K_{\mathrm{x}, \mathrm{sp}}\right)}
$$

with $\mathfrak{I m} K_{\mathrm{x}, \mathrm{sp}}>0$, and where $K_{s p}$ depends on $\omega$ and is given by the dispersion relation. $\overleftrightarrow{g}$ then features two poles at $K_{\mathrm{x}, \mathrm{sp}}$ and $-K_{\mathrm{x} \text {, sp }}$.

The residues of $\overleftrightarrow{g}$ at these poles can be calculated with $\stackrel{\leftrightarrow}{f}_{\widetilde{K_{\mathrm{x}}}}\left(K_{\mathrm{y}}, z, z^{\prime}, \omega\right)=\lim _{K_{\mathrm{x}} \rightarrow \widetilde{K_{\mathrm{x}}}}\left[\left(K_{\mathrm{x}}-\widetilde{K_{\mathrm{x}}}\right) \overleftrightarrow{g}\left(\mathbf{K}, z, z^{\prime}, \omega\right)\right]$ where $\widetilde{K_{\mathrm{x}}}$ denotes $K_{\mathrm{x} \text {, sp }}$ or $-K_{\mathrm{x}, \mathrm{sp}}$. It comes :

$$
\begin{gathered}
\stackrel{\leftrightarrow}{f}_{K_{\mathrm{x}, \mathrm{sp}}}\left(K_{\mathrm{y}}, z, z^{\prime}, \omega\right)=\frac{i}{2 K_{\mathrm{x}, \mathrm{sp}}} \frac{\gamma_{1} \epsilon_{2}}{k_{0} \sqrt{\epsilon_{1}}} \frac{\gamma_{1} \epsilon_{2}-\gamma_{2} \epsilon_{1}}{\epsilon_{1}^{2}-\epsilon_{2}^{2}} \\
\left(\hat{\mathbf{K}}^{+}-\frac{K}{\gamma_{\mathrm{m}}} \mathbf{n}_{\mathrm{m}}\right) \hat{\mathbf{p}}_{1}^{+}\left(K_{\mathrm{x}, \mathrm{sp}} \hat{\mathbf{x}}+K_{\mathrm{y}} \hat{\mathbf{y}}, \omega\right) e^{-i \gamma_{1} z^{\prime}} e^{i \gamma_{\mathrm{m}}|z|} \quad(\mathrm{A} 1 \\
\overleftrightarrow{f}_{-K_{\mathrm{x}, \mathrm{sp}}}\left(K_{\mathrm{y}}, z, z^{\prime}, \omega\right)=-\frac{i}{2 K_{\mathrm{x}, \mathrm{sp}}} \frac{\gamma_{1} \epsilon_{2}}{k_{0} \sqrt{\epsilon_{1}}} \frac{\gamma_{1} \epsilon_{2}-\gamma_{2} \epsilon_{1}}{\epsilon_{1}^{2}-\epsilon_{2}^{2}} \\
\left(\hat{\mathbf{K}}^{-}-\frac{K}{\gamma_{\mathrm{m}}} \mathbf{n}_{\mathrm{m}}\right) \hat{\mathbf{p}}_{1}^{+}\left(-K_{\mathrm{x}, \mathrm{sp}} \hat{\mathbf{x}}+K_{\mathrm{y}} \hat{\mathbf{y}}, \omega\right) e^{-i \gamma_{1} z^{\prime}} e^{i \gamma_{\mathrm{m}}|z|}
\end{gathered}
$$

where $\hat{\mathbf{K}}^{+}=\frac{K_{\mathrm{x}, \mathrm{sp}} \hat{\mathbf{x}}+K_{\mathrm{y}} \hat{\mathbf{y}}}{K_{s p}}, \hat{\mathbf{K}}^{-}=\frac{-K_{\mathrm{x}, \mathrm{sp}} \hat{\mathbf{x}}+K_{\mathrm{y}} \hat{\mathbf{y}}}{K_{s p}}$, and the other notations are defined above.

Using Eq. (7), (11), (12), (A10) and (A11), one can find the amplitudes in Eq. (13) :

$$
\begin{gathered}
E_{>}\left(K_{\mathrm{y}}, \omega, x\right)=\mu_{0} \frac{1}{2 K_{\mathrm{x}, \mathrm{sp}}} \frac{\gamma_{1} \epsilon_{2}}{k_{0} \sqrt{\epsilon_{1}}} \frac{\gamma_{1} \epsilon_{2}-\gamma_{2} \epsilon_{1}}{\epsilon_{1}^{2}-\epsilon_{2}^{2}} \\
\int_{-\infty}^{x} \mathrm{~d} x^{\prime} e^{-i K_{\mathrm{x}, \mathrm{sp}} x^{\prime}} \int \mathrm{d} y^{\prime} e^{-i K_{\mathrm{y}} y^{\prime}} \int_{-\infty}^{0} \mathrm{~d} z^{\prime} e^{-i \gamma_{1} z^{\prime}} \int \mathrm{d} t^{\prime} e^{i \omega t^{\prime}} \\
\hat{\mathbf{p}}_{1}^{+}\left(K_{\mathrm{x}, \mathrm{sp}} \hat{\mathbf{x}}+K_{\mathrm{y}} \hat{\mathbf{y}}, \omega\right) \cdot \frac{\partial \mathbf{j}}{\partial t^{\prime}}\left(\mathbf{r}^{\prime}, t^{\prime}\right) \quad(\mathrm{A} 12)
\end{gathered}
$$

$$
\begin{gathered}
E_{<}\left(K_{\mathrm{y}}, \omega, x\right)=\mu_{0} \frac{1}{2 K_{\mathrm{x}, \mathrm{sp}}} \frac{\gamma_{1} \epsilon_{2}}{k_{0} \sqrt{\epsilon_{1}}} \frac{\gamma_{1} \epsilon_{2}-\gamma_{2} \epsilon_{1}}{\epsilon_{1}^{2}-\epsilon_{2}^{2}} \\
\int_{x}^{\infty} \mathrm{d} x^{\prime} e^{-i K_{\mathrm{x}, \mathrm{sp}} x^{\prime}} \int \mathrm{d} y^{\prime} e^{-i K_{\mathrm{y}} y^{\prime}} \int_{-\infty}^{0} \mathrm{~d} z^{\prime} e^{-i \gamma_{1} z^{\prime}} \int \mathrm{d} t^{\prime} e^{i \omega t^{\prime}} \\
\hat{\mathbf{p}}_{1}^{+}\left(-K_{\mathrm{x}, \mathrm{sp}} \hat{\mathbf{x}}+K_{\mathrm{y}} \hat{\mathbf{y}}, \omega\right) \cdot \frac{\partial \mathbf{j}}{\partial t^{\prime}}\left(\mathbf{r}^{\prime}, t^{\prime}\right)
\end{gathered}
$$




\section{APPENDIX B: SURFACE PLASMON FIELD OF A DIPOLE}

The currents associated with the dipole $\mathbf{p}_{0} e^{-i \omega_{0} t}$ at a distance $d$ above the interface are given by $\mathbf{j}(\mathbf{r}, t)=$ $2 \mathfrak{R e}\left[e^{-i \omega_{0} t}(-i) \omega_{0} \mathbf{p}_{0}\right] \delta(\mathbf{r}-(-d) \hat{\mathbf{z}})$. Using the form of the surface plasmon field given by Eq. (14), one can compute the amplitude with this expression and Eq. A12) :

$$
\begin{gathered}
E_{>}\left(K_{y}, \omega\right)=-\frac{\gamma_{1} \epsilon_{2}-\gamma_{2} \epsilon_{1}}{\epsilon_{1}^{2}-\epsilon_{2}^{2}} \frac{\gamma_{1} \epsilon_{2}}{k_{0} \sqrt{\epsilon_{1}}} \frac{1}{2 K_{\mathrm{x}, \mathrm{sp}}} e^{i \gamma_{1} d} \mu_{0} \omega_{0}^{2} \\
\hat{\mathbf{p}}_{1}^{+} \cdot\left[2 \pi \delta\left(\omega-\omega_{0}\right) \mathbf{p}_{0}+2 \pi \delta\left(\omega+\omega_{0}\right) \mathbf{p}_{0}^{*}\right]
\end{gathered}
$$

Hence, using Eq. (14) and the properties $K\left(-\mathbf{K}^{*}\right)=-K^{*}(\mathbf{K}), K_{\mathrm{x}, \mathrm{sp}}\left(K_{y},-\omega\right)=-K_{\mathrm{x}, \mathrm{sp}}^{*}\left(K_{y}, \omega\right)$, $\gamma_{m}\left(K^{*},-\omega\right)=-\gamma_{m}^{*}(K, \omega), \epsilon_{m}(-\omega)=\epsilon_{m}^{*}(\omega)$ and the definition of $\hat{\mathbf{p}}_{1}^{+}$, it comes :

$$
\begin{aligned}
\mathbf{E}_{\mathrm{m}}= & -\mathfrak{R e}\left[e^{i \gamma_{m}|z|} e^{-i \omega_{0} t} \frac{\gamma_{1} \epsilon_{2}-\gamma_{2} \epsilon_{1}}{\epsilon_{1}^{2}-\epsilon_{2}^{2}} \frac{\gamma_{1} \gamma_{2}}{\epsilon_{0}}\right. \\
& \left.\int \frac{\mathrm{d} K_{y}}{2 \pi} \frac{e^{i \mathbf{K} \cdot \mathbf{r}}}{K_{\mathrm{x}, \mathrm{sp}}}\left(\hat{\mathbf{K}}-\frac{K}{\gamma_{m}} \hat{\mathbf{n}}_{m}\right)\left(\hat{\mathbf{K}}-\frac{K}{\gamma_{1}} \hat{\mathbf{z}}\right) \cdot \mathbf{p}_{0}\right]
\end{aligned}
$$

By derivating $\int \frac{\mathrm{d} K_{y}}{2 \pi} \frac{e^{i \mathbf{K} \cdot \mathbf{r}}}{K_{\mathrm{x}, \mathrm{sp}}}=\frac{1}{2} H_{0}^{(1)}\left(K_{s p} \rho\right)$ with respect to $x$ or $y$ one finds $\int \frac{\mathrm{d} K_{y}}{2 \pi} \frac{e^{i \mathbf{K} \cdot \mathbf{r}}}{K_{\mathrm{x}, \mathrm{sp}}} \hat{\mathbf{K}}=$ $\frac{i}{2} H_{1}^{(1)}\left(K_{s p} \rho\right) \hat{\rho} \quad$ and $\quad \int \frac{\mathrm{d} K_{y}}{2 \pi} \frac{e^{i \mathbf{K} \cdot \mathbf{r}}}{K_{\mathrm{x}, \mathrm{sp}}} \hat{\mathbf{K}} \hat{\mathbf{K}} \quad=$ $\frac{1}{2}\left[\left[H_{1}^{(1)}\left(K_{s p} \rho\right)\right]^{\prime} \hat{\rho} \hat{\rho}+\frac{H_{1}^{(1)}\left(K_{s p} \rho\right)}{K_{s p} \rho} \hat{\theta} \hat{\theta}\right]$. Using these relations and the value of $\mathbf{p}_{0}$, with $M(K, \omega)=$ $-\frac{\gamma_{1} \gamma_{2}}{4} \frac{\gamma_{1} \epsilon_{2}-\gamma_{2} \epsilon_{1}}{\epsilon_{1}^{2}-\epsilon_{2}^{2}}$, we get Eq.(15) and (17). According to Eq.(14), the field found in Eq.(15) and (17) only apply in the $x>0$ half-space. Using symmetries arguments, one find easily that they also apply in the $x<0$ half-space.

Eq. (15) and (17) can then be simplified using the asymptotical form of the Hankel functions $H_{n}(z) \rightarrow \sqrt{\frac{2}{\pi z}} e^{i z-\frac{1}{2} \pi i\left(n+\frac{1}{2}\right)}$. Denoting $M_{v}^{\prime}\left(K_{s p}, \omega_{0}\right)=$ $-\sqrt{\frac{2}{\pi}} e^{-i \frac{\pi}{4}} M\left(K_{s p}, \omega_{0}\right) \frac{K_{s p}}{\gamma_{1} \epsilon_{0}}$, we obtain Eq. (16). Using also the property of the Hankel functions $H_{1}^{\prime}(z)=H_{0}(z)-\frac{1}{z} H_{1}(z)$ and denoting $M_{h}^{\prime}\left(K_{s p}, \omega_{0}\right)=$ $\sqrt{\frac{2}{\pi}} e^{-i \frac{\pi}{4}} M\left(K_{s p}, \omega_{0}\right) \frac{1}{\epsilon_{0}}$, we obtain Eq. (18).

\section{ACKNOWLEDGMENTS}

This work was supported by the French National Research Agency (ANR) through Carnot Leti funding and by the French Ministry of Defense through a grant from the Direction générale de l'armement (DGA).
* Electronic address: jean-jacques.greffet@institutoptique.fr

1 R.H. Ritchie, Phys. Rev. 106, 874 (1957).

2 W. L. Barnes, A. Dereux, T.W. Ebbesen, Nature 424, 824 (2003).

3 T.W. Ebbesen, H.J. Lezec, H.F. Ghaemi, T. Thio, P.A. Wolff, Nature 391, 667 (1998).

${ }^{4}$ H. Liu, P. Lalanne, Nature 452, 06762 (2008).

5 J.C. Weeber et al., Phys. Rev. B 64, 045411 (2001).

6 S.I. Bozhevolnyi, V.S. Volkov, E. Devaux, J.Y. Laluet, T.W. Ebbesen, Nature 440, 04594 (2006).

7 M. Quinten, A. Leitner, J.R. Krenn, F.R. Ausseneg, Opt. Lett. 23, 1331 (1998).

8 Maier et al., Nature Mat. 2, 229 (2003).

9 P. Andrew, W.L.Barnes, Phys. Rev. B 64, 125405 (2001).

10 P. Anger, P. Bharadwaj and L. Novotny, Phys. Rev. Lett. 96, 113002 (2006).

11 S. Kuhn, U. Hakanson, L. Rogobete, V. Sandoghdar, Phys. Rev. Lett. 97, 017402 (2006).

12 H. Rigneault, J. Capoulade, J. Dintinger, J. Wenger, N. Bonod, E. Popov, T.W. Ebbesen, P.F. Lenne, Phys. Rev. Lett. 95, 117401 (2005).

13 J.B. Khurgin, G. Sun, R.A.Soref, Appl. Phys. Lett. 93, 021120 (2008).

14 C.E. Talley,J.B. Jackson, C. Oubre, NK Grady, CW Hollars, SM Lane, TR Huser, P. Nordlander, N.J. Halas, Nano Letters 51569 (2005)

15 E. Prodan, C. Radloff, N.J. Halas, P. Nordlander, Science 302, 419 (2003)
16 K.L. Kelly, E. Coronado, L.L. Zhao and GC Schatz J. Physical Chemistry B 107668 (2003)

17 Z. Liu, J.M. Steele, W. Srituravanich, Y. Pikus, C. Sun, X. Zhang Nanoletters 51726 (2005)

18 L. Feng, K.A. Tetz, B. Slutsky, V. Lomakin, Y. Fainman Appl.Phys.Lett. 91081101 (2007)

19 A.G. Curto, F.J. Garcia de Abajo, Nano Lett. 8, 2479 (2008)

20 N. Fang, H. Lee, C. Sun, X. Zhang, Science, 308534 (2005)

21 I. Smolyaninov, J. Elliot, A.V. Zayats, C.C. Davis, Phys. Rev. Lett. 94, 057401 (2005).

${ }^{22}$ T. Taubner, D. Korobkin, Y. Urzhunov, G. Shvets, R. Hillenbrand, Science 313, 1595 (2006).

23 T.V. Teperik, F. Garcia de Abajo, A.G. Borisov, M. Abdelsalam, P.N. Bartlett, Y. Sugawara, J.J. Baumberg, Nature Photonics 2, 299 (2008).

24 J.J. Greffet, R.Carminati, K. Joulain, J.P.Mulet, S. Mainguy, Y. Chen, Nature 416, 61 (2002).

25 F. Marquier, K. Joulain, J.P.Mulet, R. Carminati, J.J. Greffet, Y. Chen, Phys. Rev. B 69, 155412 (2004).

26 M. Laroche, C. Arnold, F. Marquier, R. Carminati, J.J. Greffet, S. Collin, N. Bardou, J.L. Pelouard, Opt. Lett. 30, 2623 (2005).

27 R. Zia, M. L Brongersma, Nature Nanotechnology 2426 (2007)

28 R.W. Alexander, G.S. Kovener, R.J. Bell, Phys. Rev. Lett. 32, 154 (1974)

29 E.T. Arakawa, M.W. Williams, R.N.Hamm, R.H.Ritchie, 
Phys. Rev. Lett. 31, 1127 (1973).

30 A. Banos, Dipole Radiation in the presence of a conducting Half-space (Pergamon, NY, 1966)

31 L. Felsen, N. Marcuwitz, Radiation and scattering of waves, (IEEE Press, NY, 1994)

32 F. Pincemin, A. Sentenac, J.J. Greffet, J. Opt. Soc. Am. A 11, 1117 (1994).

33 P. Lalanne, J.P. Hugonin, Nature Physics 2, 551 (2006).

34 I.I. Smolyaninov, C.C. Davis, J. Elliot, A.V.Zayats, Phys. Rev. Lett. 98, 209704 (2007).

35 A.Drezet, A.Hohenau, J.R. Krenn, Phys. Rev. Lett. 98, 209703 (2007).

${ }^{36}$ K. Joulain, R. Carminati, J.P.Mulet, J.J. Greffet, Phys. Rev. B 68, 245405 (2003).

37 K.Joulain, J.P. Mulet, F. Marquier, R. Carminati, J.J. Gr- effet, Surf. Sci. Rep. 57, 59 (2005).

38 A.V.Shchegrov, K. Joulain, R. Carminati, J.J. Greffet, Phys. Rev. Lett. 85, 1548 (2000).

39 R.R.Chance, A.Prock, R.Silbey, Adv. Chem. Phys. 37, 1 (1978).

40 K. Joulain, C. Henkel, Appl.Phys.B84 61 (2006)

41 J. Nelayah, M. Kociak, O. Stephan, F. Garcia de Abajo, M. Tence, L. Henrard, D. Taverna, I. Pastoriza-Santos, L. M. Liz-Marzan, C. Colliex, Nature Physics 3, 348 (2007).

42 F.J. Garcia de Abajo, M. Kociak, Phys. Rev. Lett. 100, 106804 (2008).

43 C. Chicanne et al., Phys. Rev. Lett. 88, 097402 (2002).

44 J.E. Sipe, J. Opt. Soc. Am. B 4, 481 (1987).

45 This is the case for a vacuum/metal (Drude model) interface. 